\title{
Long-term simulations of the rotational state of small irregular cometary nuclei
}

\author{
P. J. Gutiérrez ${ }^{1}$, L. Jorda ${ }^{1}$, J. L. Ortiz ${ }^{2}$, and R. Rodrigo ${ }^{2}$ \\ ${ }^{1}$ Laboratoire d'Astrophysique de Marseille, BP 8, 13376 Marseille Cedex 12, France \\ 2 Instituto de Astrofísica de Andalucía, CSIC, PO Box 3004, 18080 Granada, Spain
}

Received 27 March 2003 / Accepted 20 May 2003

\begin{abstract}
We have performed simulations of the long-term evolution of the spin state of small active comet nuclei $(1 \mathrm{~km}$ and $0.6 \mathrm{~km}$ ) in the orbit of 46P/Wirtanen under the effect of the torque of the nongravitational force. A total of 46 combinations of irregular shapes and activity patterns have been simulated. We observe typical changes of the spin period of $0.01-10 \mathrm{~h}$ at each perihelion passage during the simulations, depending on the initial spin period and on the temporal evolution of the spin state. The direction of the angular momentum also changes by 0.1 to several tens of degrees per orbit. These changes are not always associated with an observable excitation of the spin state. While the nucleus gets to excited spin states in some simulations, it remains in a pure spin state during several tens of orbits in others. Therefore, even small and very active nuclei like 46P/Wirtanen could remain in their spin state of minimum energy (pure spin) during at least several tens of perihelion passages. We find that, for the parameters used in our simulation, a drastic increase in spin period to $200 \mathrm{~h}$ is required before the excitation of the spin state can occur. Further results and their consequences for the observations of rotational parameters of cometary nuclei are thoroughly described in the text.
\end{abstract}

Key words. comets: general

\section{Introduction}

An important characteristic of the motion of comets is that it is affected by the so-called non-gravitational acceleration due to the expulsion of material from the nucleus. This was first pointed out by Bessel in the nineteenth century. In order to understand and to explain this behavior, Whipple (1950) proposed the icy conglomerate model for cometary nuclei. In his historical paper, Whipple showed that the momentum transferred to the nucleus by the sublimating ices could indeed explain the secular changes observed in the orbital elements of several comets. Whipple also suggested that if cometary nuclei were irregular bodies, the nongravitational force ${ }^{1}$ could alter the rotational state of cometary nuclei.

The discovery of two periodicities lead to the evidence that $1 \mathrm{P} /$ Halley's nucleus was in fact in a non-principal axis (hereafter "NPA" or "excited") spin state (e.g. Belton 1991). Since its nucleus is significantly larger than that of most short-period comets, several authors suggested that most comet nuclei could indeed be in excited spin states (Belton 1991; Jewitt 1992).

Send offprint requests to: P. J. Gutiérrez,

e-mail: pedro.gutierrez@oamp.fr

${ }^{1}$ Hereafter, we call "nongravitational force" or "NGF" the outgassing force due to the sublimation of ices from the surface. Other effects contribute to the overall "nongravitational force", but they are several orders of magnitude smaller (see Jorda \& Gutiérrez 2002).
The evidence that $1 \mathrm{P} /$ Halley and other comets are likely to be in NPA spin states triggered new studies of the effect of the torque of the NGF on spin states. Samarasinha et al. (1986), using an approximate description, obtained the timescale for change the spin rate or the angular momentum of a comet nucleus. This timescale is given by the relationship:

$\tau \approx \frac{\omega}{\dot{\omega}} \approx \frac{L}{\mathrm{~d} L / \mathrm{d} t} \approx \frac{8 \pi^{2} \alpha \rho r_{n}^{5}}{3 \eta \mathrm{d} r_{n} \dot{m} v P_{\mathrm{rot}}}$

where $\alpha$ is a ratio between the actual moment of inertia and the moment of inertia of a spherical body with the same mean radius, $r_{n}, d r_{n}$ is a characteristic moment arm, $\eta$ is a global momentum transfer coefficient, $\dot{m}$ is the mass loss rate due to sublimation, $v$ is the gas velocity, and $P_{\text {rot }}$ is the nucleus spin period. Samarasinha et al. (1986) showed that for comet 1P/Halley this timescale was comparable to the orbital period and that, therefore, it was very likely that $1 \mathrm{P} /$ Halley's spin period had changed since its 1910 apparition.

Several authors have investigated the generation and stability of NPA spin states (e.g., Wilhelm 1987; Julian 1988; Peale \& Lissauer 1989). All of them obtained similar results, confirming that the momentum transferred to the nucleus by the sublimating ice could modify the angular momentum in a single orbital step. Peale \& Lissauer (1989) also pointed out that complex rotation easily appears only for nearly symmetric tops. More recently, Samarasinha \& Belton (1995) 


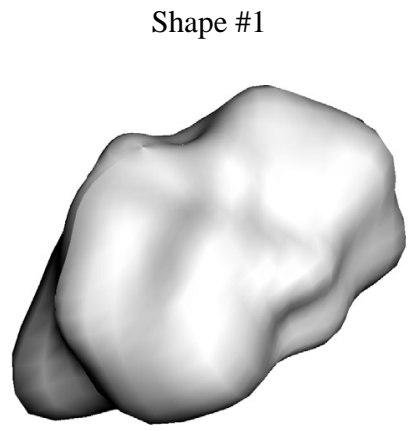

Shape \#3

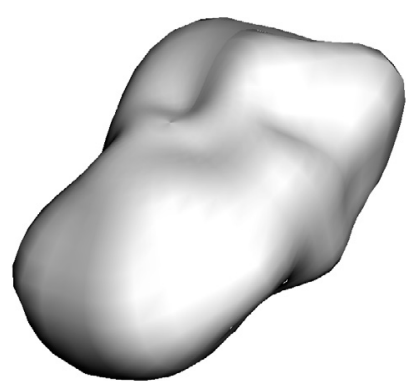

Shape \#2

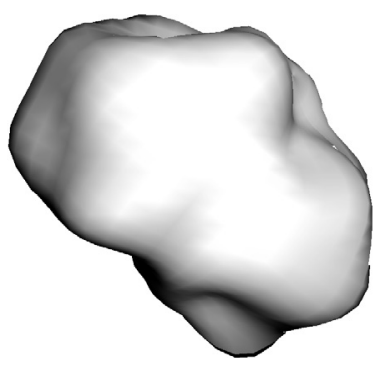

Shape \#4

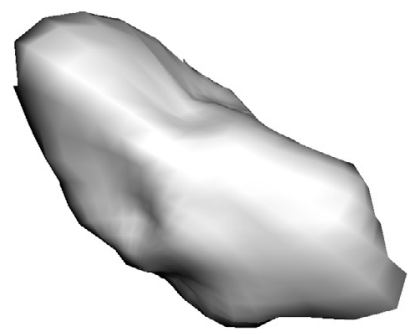

Fig. 1. Irregular shapes used in the simulations. The moments of inertia $\left(I_{x}: I_{y}: I_{z}\right)$ are normalized by mass $\cdot$ radius $\left.^{2}: 1\right) 0.32: 0.56: 0.61$, 2) 0.40:0.51:0.59, 3) 0.42:0.64:0.70., and 4) 0.46:1.25:1.36.

extensively studied the long-term evolution of the rotational state of Halley-like nuclei under the effect of the sublimationinduced torque. They considered a body with several active areas on its surface initially rotating with a spin period of 10 days. From their study, they concluded that for acceptable locations of the active regions, the nucleus can be rotating in a "stable" complex state over many orbits. They also confirmed that a significant fraction of cometary nuclei could be rotating in NPA rotational states. Samarasinha et al. (1996) also studied the long-term rotational evolution of a simulated nucleus in the orbit of comet 46P/Wirtanen. From their study, where they also used a large prolate body, they concluded that 46P/Wirtanen is very likely to be in an excited rotational state and that rapid changes in the rotational state are possible. Very recently, Neishtadt et al. (2002) also studied the evolution of comet nucleus rotation. They conclude that comets tend to gain angular momentum over time and that the fraction of active surface area on a comet may lead to certain values of nutation angle and cause its angular momentum to align in specific directions related to its perihelion. However, most of these simulations have been carried out using prolate or nearly prolate bodies, and production rates evaluated with Marsden et al.'s (1973) relationship.

Keller et al. (2000) and Szegö et al. (2001) used a different approach. They used an irregular shape to represent the nucleus and a thermophysical model to calculate the sublimation rate and the torque acting on the uniformly active nucleus. Using a similar approach, Gutiérrez et al. (2002) (henceforth Paper I) also studied the rotational evolution of several irregular cometary nuclei under different initial conditions, but only for one orbital period. The main result of these works is that, for the initial conditions considered, large changes in the spin period and in the spin axis orientation are expected for small cometary nuclei rotating very fast $(6 \mathrm{~h})$. Nevertheless, during a single orbital step and for nuclei rotating very fast, the torque is too small to put the nucleus into a NPA spin state.

As an extension of our previous work (Paper I) we have performed simulations with the three same irregular shapes plus a new one, and the same activity patterns, in order to study the long-term evolution of the spin state of small irregular cometary nuclei. In the next section, the thermophysical model used in the simulations is briefly described. For a detailed description see Gutiérrez et al. (2001, 2002).

\section{Modeling}

\subsection{Numerical solution of the Euler equations}

The nucleus is assumed to be an irregularly-shaped body separated into 1520 surface elements, or "cells". Three of the four irregular shapes and the activity patterns on the surface are generated with Gaussian random shapes (Peltoniemi et al. 1989; Muinonen 1998). The fourth irregular shape is generated by removing and adding randomly distributed sections of spheres to an ellipsoid, and by smoothing the resulting surface model. Figure 1 shows the irregular shapes and Fig. 2 the activity patterns. The coordinates of the center of mass and the three moments of inertia $I_{x}<I_{y}<I_{z}$ associated to the principal axes are calculated assuming a uniform density of $500 \mathrm{~kg} / \mathrm{m}^{3}$. This density is close to the mean value estimated by Rickman (1989) for comet Halley.

We use a simplified surface thermal model to compute the net sublimation rate and surface temperature. In this model, 

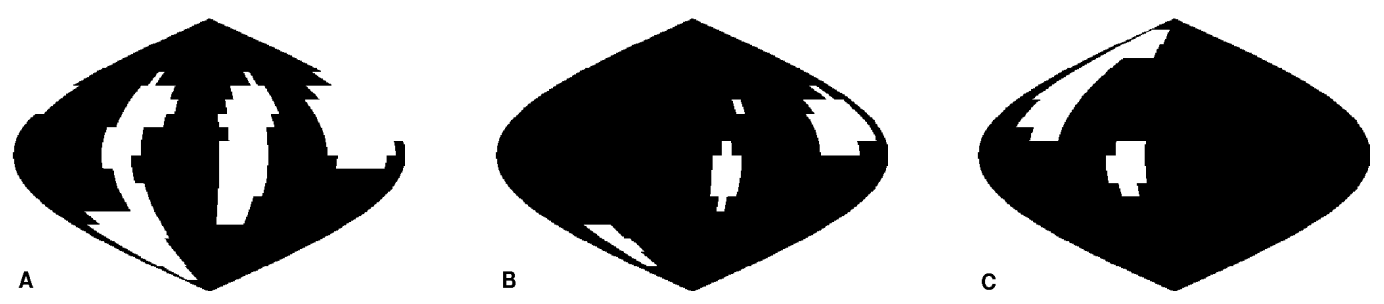

Fig. 2. Besides the uniformly active case, the distributions of activity shown in the figure have been used in this study. In these sinusoidal projections, white regions consist of water ice and black regions represent the non-sublimating inactive areas. The white regions represents, approximately $30 \% \mathbf{A}), 10 \% \mathbf{B}$ ) and $9 \%$ C) of the total surface area of the objects shown in Fig. 1.

Table 1. Physical parameters used in the simulations.

\begin{tabular}{lcl}
\hline \hline Density & $\rho$ & $500 \mathrm{~kg} / \mathrm{m}^{3}$ \\
Albedo & $A$ & 0.04 \\
Emissivity & $\epsilon$ & 0.96 \\
Latent Heat & $L$ & $48600 \mathrm{~J} / \mathrm{mol}$ \\
Condensation & $\alpha$ & 0.25 \\
Mom. Trans. Coef. & $\eta$ & 0.5 \\
\hline
\end{tabular}

water is the only volatile constituent, and the energy balance equation at the surface is given by:

$\frac{F_{0}\left(1-A_{i}\right) c_{i}}{r_{\mathrm{h}}^{2}} \cos \left(\theta_{i}\right)=\epsilon \sigma T_{i}^{4}+L(1-\alpha) Z_{\mathrm{HK}}\left(T_{i}\right)$

where $i$ stands for the cell number, $F_{0}$ is the solar constant, $A$ the Bond albedo, $r_{\mathrm{h}}$ the heliocentric distance, $\theta_{i}$ the solar zenith angle, and $c_{i}$ is 1 if the cell is illuminated and 0 otherwise (taking shadowing effects into account). The parameter $\epsilon$ is the infrared emissivity, $\sigma$ Stefan-Boltzman's constant, $L$ is the latent heat of sublimation of water, and $T_{i}$ is the cell surface temperature. In the previous equation, $Z_{\mathrm{HK}}$ represents the Hertz-Knudsen water sublimation rate, which is multiplied by the factor $(1-\alpha)$ to take into account the recondensation of backscattered water molecules (Crifo 1987). In Table 1, the values of the physical parameters used in the simulations are summarized.

The torque acting on the nucleus follows the relationship:

$\boldsymbol{N}_{\text {nongrav }}=-m \sum_{i=1}^{N} Z_{H K} S_{i} \eta v_{g, i} \boldsymbol{r}_{i} \times \boldsymbol{n}_{i}$

where the summation is over the $N$ illuminated active cells, $m$ is the mass of water molecules, $S_{i}$ is the area of cell number $i$, $\boldsymbol{r}$ is the radial vector joining the center of mass of the body and the center of the cell, and $\boldsymbol{n}$ is the vector normal to the surface. In the previous expression, $v_{\mathrm{g}, i}$ is the mean gas velocity at temperature $T_{i}$, and $\eta$ is the momentum transfer coefficient. In this study we assume $\eta=0.5$ (Crifo 1987), which is also the value used by Rickman (1989) in order to estimate the density for comet Halley ${ }^{2}$.

We solve numerically the Euler equations (Landau \& Lifshitz 1976), using the torque calculated from Eq. (3) and the

\footnotetext{
${ }^{2}$ It is necessary to note that recently, higher values for $\eta(\sim 0.9)$ have been re-computed by introducing the static pressure into the "rocket equation" (Rodionov et al. 2002).
}

moments of inertia of the bodies, in order to obtain the temporal evolution of the angular velocities $\Omega_{x}, \Omega_{y}$ and $\Omega_{z}$, and that of the three Euler angles $\phi, \theta$ and $\psi$. A Burlish-Stoer algorithm with adaptive time step is used to integrate the differential equations. The initial time step is $\Delta t / 100$, where $\Delta t$ is a numerical parameter of the code. At $t+\Delta t$, the torque is calculated again. It means that from $t$ to $t+\Delta t$, the torque is considered constant, although the Euler equations are solved in a large number of points between $t$ and $t+\Delta t$. In principle, this is a reasonable approximation if $\Delta t$ is small enough. In the code, $\Delta t=\min \left[175 \mathrm{~s}, P_{\text {rot }}[\mathrm{s}] / 250\right]$, i.e., the torque is calculated at least 250 times per rotation period, even if the rotation period changes during the simulation. We have checked the code comparing the solution obtained with the numerical integrator with analytical solutions for prolate bodies subject to a time-dependent torque. The relative errors in the Euler angles and in their velocities were always lower than $10^{-6}$. The details of the numerical procedure, the expressions of the differential equations in terms of different sets of Euler angles and the tests we have done to check the numerical integrator have been thoroughly described in Gutiérrez (2001).

\subsection{Simulation strategy}

Simulations have been performed for the orbit of comet 46P/Wirtanen (Marsden \& Williams 1999), currently (Mar. 2003) one of the two possible targets for ESA's Rosetta mission. Nevertheless, our main goal is to study the rotational evolution of a small nucleus on the orbit of a short period comet under a large number of different initial conditions (mostly shape, activity pattern and spin period). In principle, it is expected that some effects can be scaled to larger comet nuclei.

A total of 46 simulations using the shapes of Fig. 1, scaled to mean radii of 1 and $0.6 \mathrm{~km}$ (Lamy et al. 1997), with the activity patterns shown in Fig. 2, including the case of a uniformly active nucleus, have been carried out.

The total water production rate at a given time can be calculated by co-adding all the elementary production rates $\delta Q_{i}=(1-\alpha) Z_{\mathrm{HK}}\left(T_{i}\right) S_{i}$ of the illuminated active cells. We have compared the water production rate obtained with our simulated nuclei with the water production rate measured by Bertaux et al. (1999) for comet 46P/Wirtanen. The water production rate of all our uniformly active nuclei at perihelion is slightly larger than the observed one and the active surface fraction required to match the observed production rate ranges 


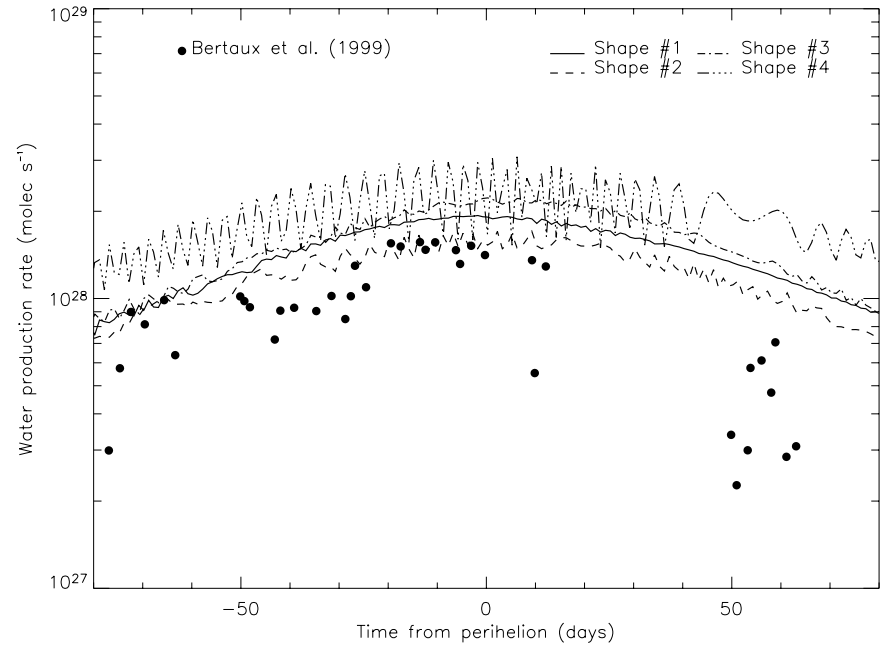

Fig. 3. Water production rate of comet $46 \mathrm{P} /$ Wirtanen measured by Bertaux et al. (1999). In this plot, water production rates obtained with the thermophysical model described in the text are also shown for the different irregular shapes used in this study. The shapes were scaled to a mean radius of $600 \mathrm{~m}$ and considered as uniformly active nuclei.

between 0.5 and 1 , a value in agreement with previous calculations (Fink et al. 1998; Rickman \& Jorda 1998). This is illustrated in Fig. 3. We feel that it makes no sense to intend to go beyond that, and to try to reproduce the observational water production rate since the actual shape and spin axis orientation are unknown, and the measurements are affected by uncertainties mainly due to the model used to retrieve production rates from column densities (e.g., Rickman \& Jorda 1998).

The initial spin axis orientation has been set to $I=45^{\circ}$ and $\Phi=60^{\circ}$, where $I$ is the obliquity of the spin axis, and $\Phi$ is the argument of the subsolar point at perihelion (Sekanina 1981a). In all the simulations, the nucleus initially rotates around its shortest principal axis, in its lowest rotational energy level for its angular momentum. Three initial spin periods have been used: $6 \mathrm{~h}$ (Lamy et al. 1997), and 48 and $240 \mathrm{~h}$ as used by Samarasinha et al. (1996).

Most of the simulations cover a period of at least 10 orbits. Some of them cover a much larger period, up to 100 orbits and only three of them where interrupted before 10 orbits because the spin period became lower than $1 \mathrm{~h}$ in a few orbits after starting or they showed a very stable rotational evolution.

\section{Results and discussion}

Table 2 summarizes the initial conditions of the simulations and the final mean spin period and rotational state after a number of orbits. In the following, we extend the definition of the spin period to the quantity $2 \pi / \sqrt{\Omega_{x}^{2}+\Omega_{y}^{2}+\Omega_{x}^{2}}$, where $\Omega_{x}$, $\Omega_{y}$, and $\Omega_{x}$ are the components of the angular velocities in the body-fixed reference system. As in Paper I, we also define the normalized rotational excitation level by the quantity $\left(1-L^{2} / 2 E I_{z}\right) /\left(1-I_{x} / I_{z}\right)$, where $L$ is the modulus of the angular momentum and $E$ is the rotational energy. The abovedefined excitation level is equal to zero when the nucleus rotates around the principal axis of lowest energy (small axis), and is equal to one when it rotates around its principal axis of highest energy (long axis).

We observe two main effects in the simulations. First of all, the direction of the angular momentum and the value of the spin period vary with the timescale of Eq. (1). Second, NPA (excited) spin states appear in some cases. In the following sections, we give a detailed description of these phenomena.

\subsection{Temporal evolution of the spin state}

One of the main results of the simulations is that in some cases the nucleus remains very close to its initial PA spin state (of lowest energy) until the end of the simulation. In Table 2, these cases are marked with $\circ$ in the last column. For all of them, the initial spin period is $6 \mathrm{~h}$ and usually decreases at each orbital step, preventing the nucleus from reaching an excited spin state (see Fig. 4, simulation \#7).

In other simulations, marked with o in Table 2, the nucleus undergoes an excitation in one of the first orbits. After that, the nucleus returns to the PA state of lowest rotational energy. In these simulations, the nucleus spins down during the first orbits, which leads to a NPA state in which the three angular velocities have comparable values. After this temporary excitation, the component of the angular velocity around the shortest axis changes of sign and its absolute value increases again rapidly: the nucleus comes back close to its initial PA state of lowest energy (see Fig. 5, simulation \#3).

In other simulations, marked with $\bullet$ in Table 2 , the nucleus also spins down during the first orbits. However, it then reaches a PA state of highest rotational energy after a few orbits (Fig. 6, simulation \#15).

Finally, in other simulations, marked with $\diamond$ and with $\star$, the nucleus is excited and remains rotating in a NPA state during the rest of the simulation. In the simulations marked with $\star$, the rotational excitation level changes slowly at each perihelion passage. On the contrary, in the simulations marked with $\diamond$, the nucleus suffers large changes of the rotational excitation level at each perihelion passage. The latter case corresponds to the largest final spin period. In Fig. 7, the evolution of the rotational excitation levels for simulations \#21 and \#27 is shown.

For prolate bodies in the PA state of lowest energy, the timescale for excitation is that of the Eq. (1) (Jewitt 1992). However, there is no simple way of estimating the excitation timescale for bodies with three different moments of inertia. In our numerical simulations, we observe that starting from the lowest rotational energy level and before the excitation takes place, the spin period increases significantly, i.e. it seems that the the spin period has to be above a certain threshold before the nucleus can get significantly excited. If the initial spin period is below this threshold, changes of the spin period must therefore occur (it does with the timescale of the relationship (1)) before the torque of the NGF can start to put the nucleus into a NPA spin state. In our simulations, spin periods just before the nucleus can get significantly excited are larger than $200 \mathrm{~h}$. However, this value certainly depends on several parameters (nucleus size, production rate, etc.). 
Table 2. Results of the long-term simulations.

\begin{tabular}{|c|c|c|c|c|c|c|c|c|}
\hline $\begin{array}{c}\text { Sim. } \\
\# \\
\end{array}$ & $\begin{array}{l}\text { Shape }^{a} \\
\text { nat }\end{array}$ & $\begin{array}{c}\text { Act. Frac. }{ }^{b} \\
(\%)\end{array}$ & $\begin{array}{c}\text { Radius }^{c} \\
(\mathrm{~m})\end{array}$ & $\begin{array}{r}P_{\text {init }}{ }^{d} \\
(\mathrm{hrs}) \\
\end{array}$ & $\begin{array}{c}P_{\text {final }}{ }^{e} \\
\text { (hrs) }\end{array}$ & $\begin{array}{l}\text { Final }^{f} \\
\text { state }\end{array}$ & $\begin{array}{c}\begin{array}{c}\text { Number of } \\
\text { orbits }\end{array} \\
\end{array}$ & Key \\
\hline$\# 1$ & 1 & $\operatorname{Unif}(100)$ & 600 & 6 & 1.8 & $E_{\min }$ & 20 & $\dot{0}$ \\
\hline \#2 & 1 & Unif(100) & 1000 & 6 & 3.5 & $E_{\min }$ & 12 & 。 \\
\hline \#3 & 1 & A (30) & 600 & 6 & $<1$ & $E_{\min }$ & 11 & o \\
\hline \#4 & 1 & A (30) & 1000 & 6 & 5 & $E_{\max }$ & 10 & - \\
\hline$\# 5$ & 1 & B (10) & 600 & 6 & 1.2 & SAM & 78 & $\star$ \\
\hline \#6 & 1 & B (10) & 1000 & 6 & 3.2 & $E_{\min }$ & 13 & $\circ$ \\
\hline \#7 & 1 & C (10) & 600 & 6 & 1.5 & $E_{\min }$ & 20 & $\circ$ \\
\hline \#8 & 1 & C (10) & 1000 & 6 & 4.2 & $E_{\min }$ & 11 & $\circ$ \\
\hline \#9 & 2 & Unif(100) & 600 & 6 & 40 & LAM & 20 & $\diamond$ \\
\hline$\# 10$ & 2 & Unif(100) & 1000 & 6 & 2.8 & $E_{\min }$ & 11 & $\circ$ \\
\hline$\# 11$ & 2 & A (30) & 600 & 6 & $<1$ & $E_{\max }$ & 6 & - \\
\hline$\# 12$ & 2 & A (30) & 1000 & 6 & 1.3 & $E_{\max }$ & 11 & - \\
\hline \#13 & 2 & B (10) & 600 & 6 & 1.1 & $E_{\min }$ & 10 & o \\
\hline \#14 & 2 & B (10) & 1000 & 6 & 1.6 & $E_{\min }$ & 19 & o \\
\hline \#15 & 2 & C (10) & 600 & 6 & 1.1 & $E_{\max }$ & 15 & - \\
\hline$\# 16$ & 2 & $\mathrm{C}(10)$ & 1000 & 6 & 3.6 & $E_{\max }$ & 19 & - \\
\hline$\# 17$ & 3 & Unif(100) & 600 & 6 & 1.8 & SAM & 40 & $\star$ \\
\hline$\# 18$ & 3 & Unif(100) & 1000 & 6 & 5.6 & $E_{\min }$ & 12 & $\circ$ \\
\hline$\# 19$ & 4 & Unif(100) & 600 & 6 & 4 & $E_{\min }$ & 12 & $\circ$ \\
\hline$\# 20$ & 4 & Unif(100) & 1000 & 6 & 5.7 & $E_{\min }$ & 13 & $\circ$ \\
\hline \#21 & 1 & Unif(100) & 600 & 48 & 14 & LAM & 52 & $\star$ \\
\hline \#22 & 1 & Unif(100) & 1000 & 48 & 16.2 & SAM & 17 & $\diamond$ \\
\hline$\# 23$ & 2 & Unif(100) & 600 & 48 & 5.3 & LAM & 80 & $\star$ \\
\hline \#24 & 2 & Unif(100) & 1000 & 48 & 8 & $E_{\max }$ & 36 & $\star$ \\
\hline$\# 25$ & 3 & Unif(100) & 600 & 48 & 6.2 & SAM & 33 & $\star$ \\
\hline \#26 & 3 & Unif(100) & 1000 & 48 & 12.8 & SAM & 13 & $\star$ \\
\hline \#27 & 4 & Unif(100) & 600 & 48 & 21.3 & SAM & 20 & $\diamond$ \\
\hline$\# 28$ & 4 & Unif(100) & 1000 & 48 & 25 & LAM & 9 & $\star$ \\
\hline$\# 29$ & 1 & Unif(100) & 600 & 240 & 4.5 & LAM & 100 & $\star$ \\
\hline$\# 30$ & 1 & Unif(100) & 1000 & 240 & 16.5 & LAM & 44 & $\star$ \\
\hline$\# 31$ & 1 & A (30) & 600 & 240 & $<1$ & $E_{\min }$ & 11 & o \\
\hline \#32 & 1 & A (30) & 1000 & 240 & 2.6 & $E_{\min }$ & 11 & o \\
\hline \#33 & 1 & B (10) & 600 & 240 & 1 & LAM & 34 & $\star$ \\
\hline \#34 & 1 & B (10) & 1000 & 240 & 1.8 & $E_{\max }$ & 10 & - \\
\hline \#35 & 1 & C (10) & 600 & 240 & $<1$ & $E_{\max }$ & 11 & - \\
\hline \#36 & 1 & $\mathrm{C}(10)$ & 1000 & 240 & 1.4 & $E_{\max }$ & 4 & - \\
\hline \#37 & 2 & Unif(100) & 600 & 240 & 6 & LAM & 30 & $\star$ \\
\hline \#38 & 2 & Unif(100) & 1000 & 240 & 4.9 & LAM & 39 & - \\
\hline$\# 39$ & 2 & A (30) & 600 & 240 & $<1$ & $E_{\max }$ & 4 & • \\
\hline \#40 & 2 & A (30) & 1000 & 240 & $<1$ & $E_{\max }$ & 12 & - \\
\hline \#41 & 2 & B (10) & 600 & 240 & 1.3 & $E_{\min }$ & 8 & $\dot{0}$ \\
\hline \#42 & 2 & B (10) & 1000 & 240 & 1.8 & $E_{\min }$ & 16 & ¿ \\
\hline \#43 & 2 & C (10) & 600 & 240 & 2 & $E_{\max }$ & 10 & - \\
\hline \#44 & 2 & C (10) & 1000 & 240 & 2 & $E_{\max }$ & 16 & - \\
\hline \#45 & 3 & Unif(100) & 600 & 240 & 2.98 & LAM & 67 & $\star$ \\
\hline \#46 & 3 & Unif(100) & 1000 & 240 & 15 & SAM & 20 & $\diamond$ \\
\hline
\end{tabular}

${ }^{a}$ Shape number. ${ }^{b}$ Active pattern as shown in Fig. 2 (Overall surface active fraction). ${ }^{c}$ Mean radius of the nucleus. ${ }^{d}$ Initial spin period (principal axis rotation is assumed). ${ }^{e}$ Mean "spin period" after the number of orbits indicated in the 8th column. ${ }^{f}$ Rotational state after the number of orbits indicated in the 8th column.

We performed additional simulations in order to better understand the excitation process. We performed simulations around the time when the excitation starts for simulation \#41. This simulation is specially appropriate for such a study because the components of the torque along the shortest and largest principal axes of inertia have a "simple" behavior, with only one maximum. In this simulation, the nucleus initially rotates with a period of 10 days. In the pre-perihelion branch of the first orbit, the angular velocity $\Omega_{z}$ decreases, and the nucleus then reaches a NPA spin state. From this point on, the angular velocity $\Omega_{x}$ starts to increase very fast, leading the nucleus to rotate close to the PA state of highest energy until the end of the simulation.
We solve the Euler equations around the time of the excitation (orbit \#1) using a time-averaged torque $N$ of the NGF, but we multiply $N_{x}$ by a factor less than one until the nucleus, after a temporary excitation, comes back to its PA state of lowest energy.

We empirically find that when the nucleus gets excited, it remains rotating in an excited state only when the coupling term in the Euler equation describing the evolution of $\Omega_{z}$ becomes greater than $N_{z}$, while the corresponding two terms describing the evolution of $\Omega_{x}$ are of the same order. This translates into the following relationships:

$$
\frac{\left|N_{x}\right|}{\left|\left(I_{z}-I_{y}\right) \Omega_{y} \Omega_{z}\right|} \approx 1 \quad \text { and } \quad \frac{\left|N_{z}\right|}{\left|\left(I_{y}-I_{x}\right) \Omega_{y} \Omega_{x}\right|}<1 .
$$



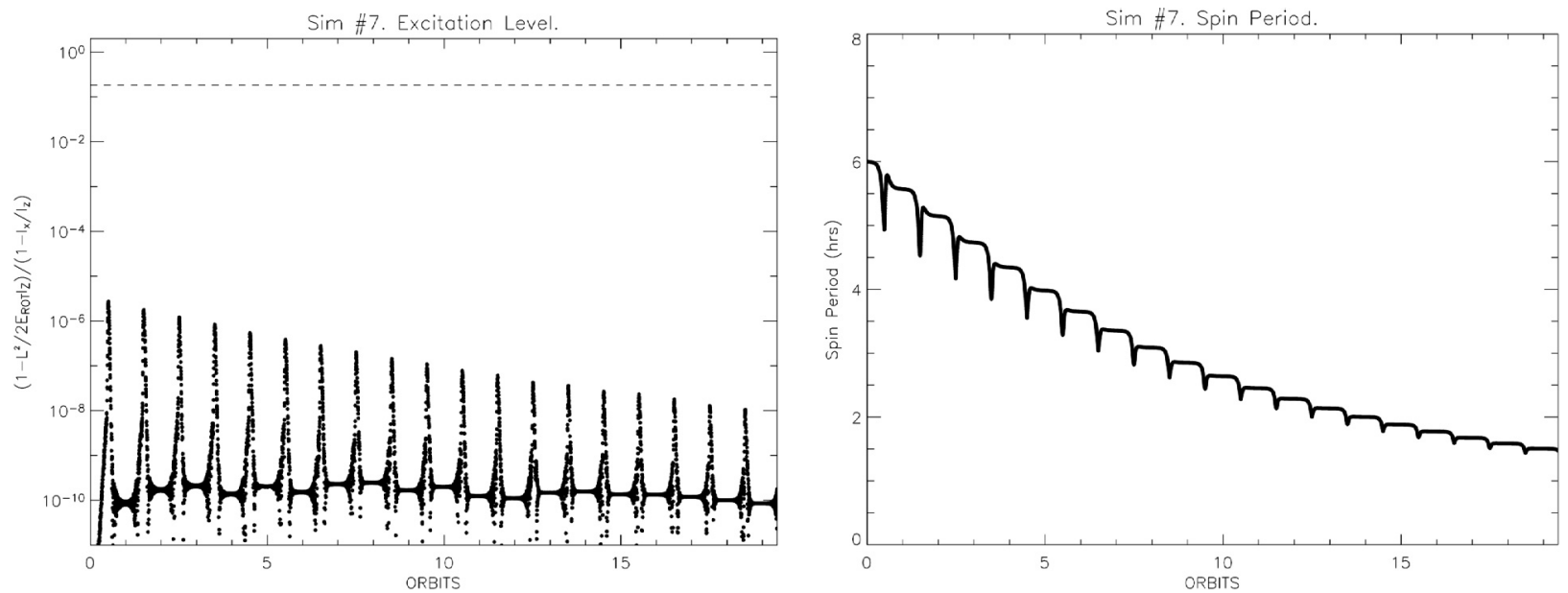

Fig. 4. Evolution of the "excitation level" (left) and of the spin period (right) for simulation \#7 (initial spin period of $6 \mathrm{~h}$ ). In the left plot, the dashed line marks the separation level between SAM and LAM modes. A slight excitation $\left(<10^{-5}\right)$ appears periodically near perihelion $($ left plot): strictly speaking, the nucleus is rotating in a SAM mode but from a practical point of view, this NPA state is indistinguishable from a PA state.
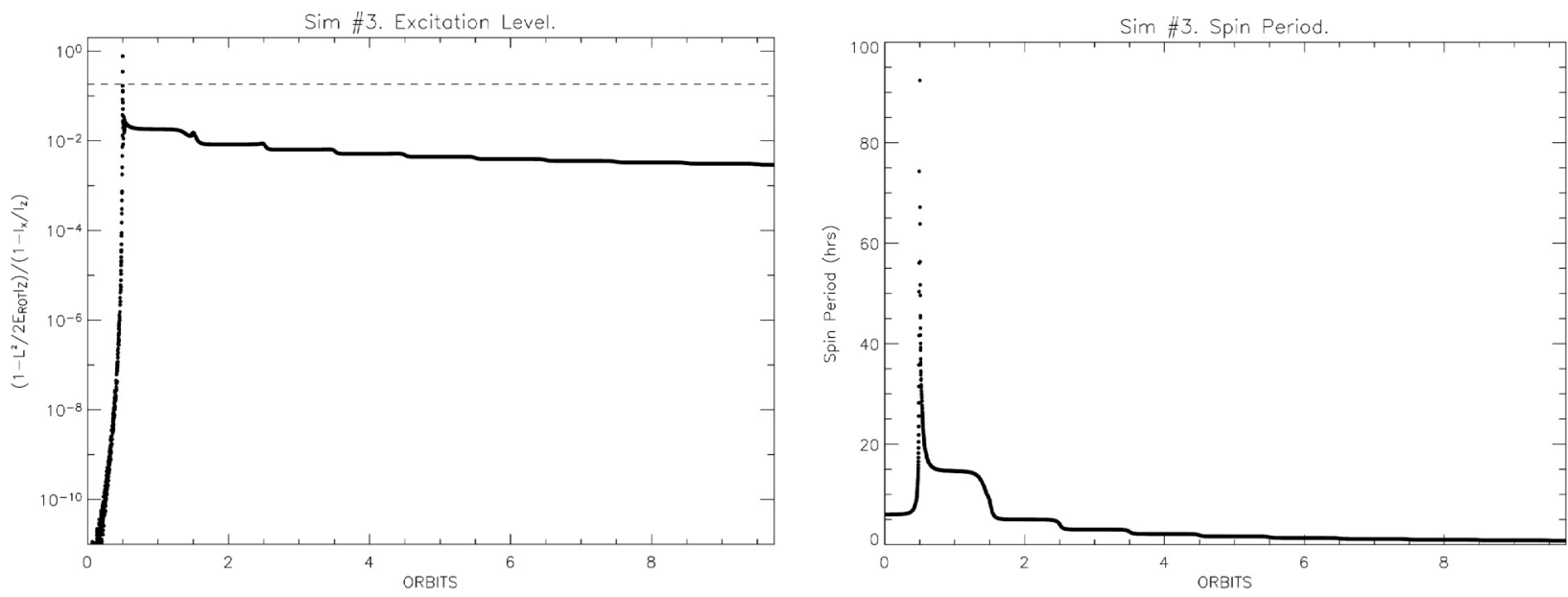

Fig. 5. Evolution of the "excitation level" (left) and of the spin period (right) for simulation \#3 (initial spin period of $6 \mathrm{~h}$ ). In the left plot, the dashed line marks the separation between SAM and LAM modes. In this simulation, the maximum mean spin period reached in the first orbit is $272 \mathrm{~h}$.
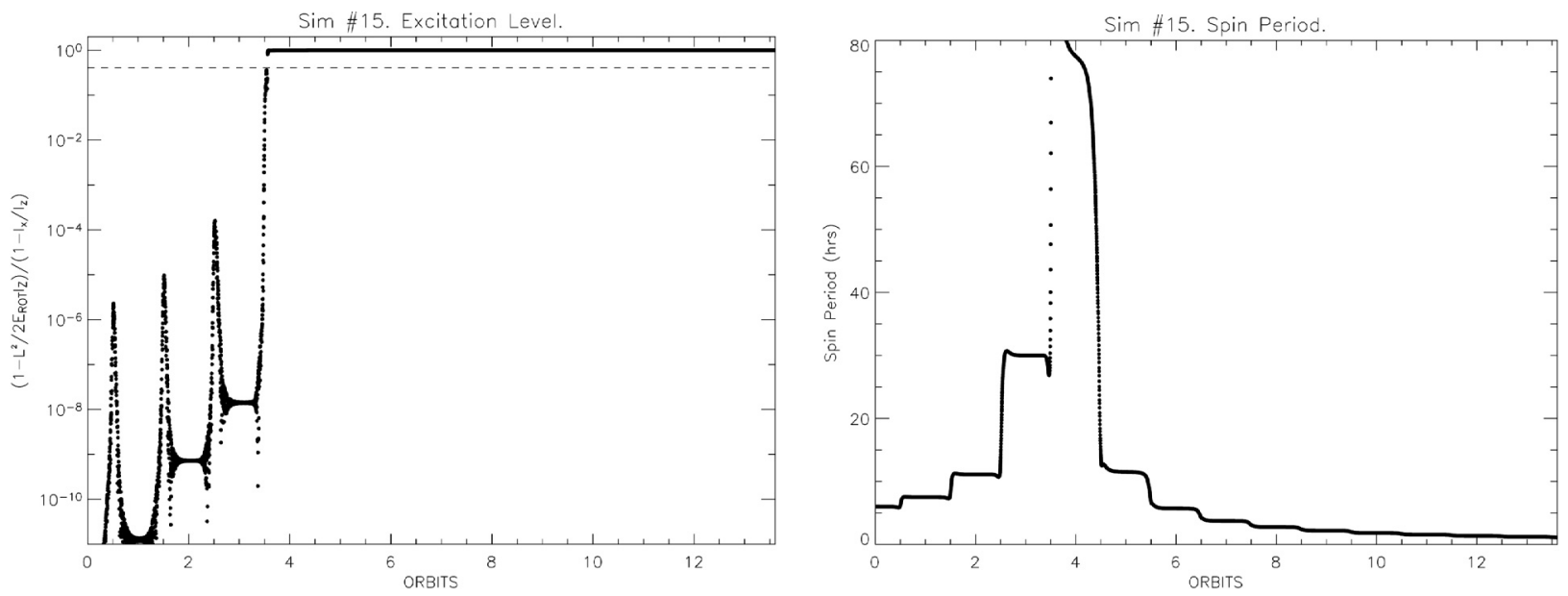

Fig. 6. Evolution of the normalized "excitation level" (left) and of the spin period (right) for simulation \#15 (initial spin period of 6 h). In the plot on the left, dashed line marks the separation level between SAM and LAM modes. In this simulation, the maximum mean spin period reached in the fourth orbit is 24.4 days. 

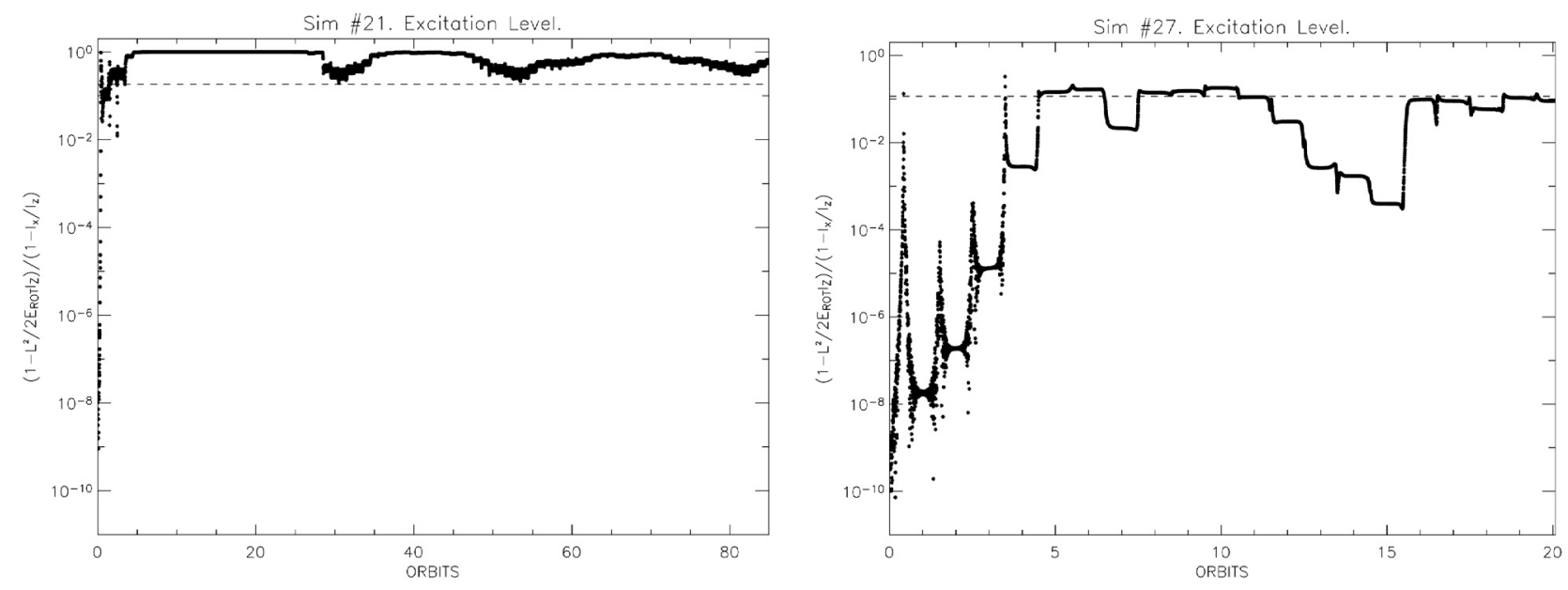

Fig. 7. Rotational excitation level for simulations \#21 (left) and \#27 (right).

This corresponds to the following condition on the ratio between the two components $N_{x}$ and $N_{y}$ of the torque:

$\frac{\left|N_{x}\right|}{\left|N_{z}\right|}>\frac{\left(I_{z}-I_{y}\right)}{\left(I_{y}-I_{x}\right)} \frac{\left|\Omega_{z}\right|}{\left|\Omega_{x}\right|}=\xi \frac{\left|\Omega_{z}\right|}{\left|\Omega_{x}\right|}$.

Equation (5) can be seen as a condition required but not sufficient for the nucleus to remain rotating in NPA state. When the previous expression is not verified, the nucleus, after a temporary excitation, comes back to the PA state of lowest energy.

In the simulations, when the nucleus starts to rotate in a NPA state, the previous condition is always verified. Nevertheless, in many simulations, a few days after this "temporary excitation", the torque components act in the opposite direction, i.e., $N_{x}$ tends to decrease $\Omega_{x}$ and $N_{z}$ tends to increase $\Omega_{z}$. The final result is that the nucleus comes back to its PA state of lowest energy, although Eq. (5) was satisfied at some point of the orbit.

The previous condition also indicates that, for particular torque components and moments of inertia, a critical period exists, as it was pointed out before. When the spin period is above this critical period, the nucleus remains rotating in an excited state. In our simulations, $\left|N_{x}\right|$ is generally of the same order than $\left|N_{z}\right|$, while $\left|\Omega_{z}\right|>>\left|\Omega_{x}\right|$ when the nucleus rotates close to the PA state of lowest energy (pure spin). This explains why an increase of the spin period (a decrease of $\left|\Omega_{z}\right|$ ) is required before the excitation can occur.

The condition of Eq. (5) also explains why quasi-prolate bodies are more easily excited than irregular ones (Peale \& Lissauer 1989). For such bodies, $\xi \sim 0$, and NPA spin states can appear even when $\left|\Omega_{x}\right|<<\left|\Omega_{z}\right|$ and $\left|N_{x}\right| \sim\left|N_{z}\right|$. The only cases where NPA spin states do not occur are those for which $N_{x}$ is close to zero, as already noticed by Samarasinha \& Belton (1995).

We can observe in Table 2 that the long-term evolution of the spin state is different for different activity patterns. Uniformly active objects in NPA spin states usually do not reach the PA state of maximum energy, unlike objects with reduced activity patterns. Let us consider the extreme case of a nucleus with a constant direction of the NGF near perihelion (in the model adopted here, this means only one active cell). The effect of the torque on the three angular velocities $\Omega_{i}$ is very similar during all the simulation, even if the direction of the angular momentum changes. In particular, if the torque causes the angular velocity along the largest axis of inertia to increase, it will continue to do so until the stable PA spin state of maximum energy is reached ${ }^{3}$. As we have considered small nuclei, this process is very fast (see Fig. 6). It would be obviously slower for larger or less active bodies. In the opposite case of a uniformly active nucleus, the three components of the torque have a "random" behavior from an orbit to the next one. The effect of the torque on $\Omega_{i}$ during one or several orbits can be partially compensated during the next orbital step(s). This leads to the "random evolution" of the excitation level observed in some simulations (see Fig. 7).

\subsection{Temporal evolution of the spin period}

The spin period suffers significant changes at every perihelion passage. These changes range from a few minutes, when the nucleus is rotating with a period lower than $6 \mathrm{~h}$ in a PA state (both of largest and of lowest energy) to several tens of hours when the nucleus is rotating very slowly in a NPA state. The changes usually occur \pm 150 days around perihelion and can be very fast, especially for nuclei with small active areas and an initial spin period of $240 \mathrm{~h}$ (e.g., simulations \#35 and \#44). For example, in simulation \#44, in the second orbit, the spin period decreases from approximately $30 \mathrm{~h}$ to $10 \mathrm{~h}$. In this case, the spin period decreases from $20 \mathrm{~h}$ to $17.4 \mathrm{~h}$ from day -100 (at $r_{\mathrm{h}}=1.64 \mathrm{AU}$ for the orbit of comet 46P/Wirtanen) to day -79 $\left(r_{\mathrm{h}}=1.44 \mathrm{AU}\right)$. The changes are particularly fast and large just before the nucleus gets excited. This can be seen, for example, in simulation \#15 (right plot of Fig. 6). In this case, the spin period drastically changes during the fourth orbit, just before the nucleus gets excited.

When the nucleus is rotating in a NPA spin state, the spin period is usually larger than $10 \mathrm{~h}$ and the changes at each orbital step are frequently larger than $1 \mathrm{~h}$. Nevertheless, there are some cases for which the nucleus is rotating with a relatively

\footnotetext{
3 Although not necessarily in a linear way if the modulus of the NGF changes.
} 


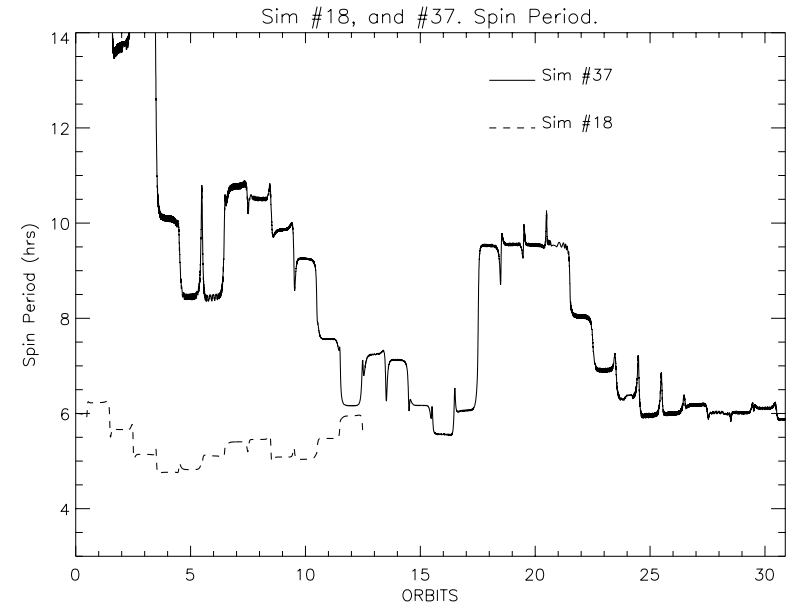

Fig. 8. Evolution of the spin period for simulations \#18 and \#37. Simulation \#18 corresponds to a nucleus initially rotating with a spin period of $6 \mathrm{~h}$. The nucleus remains in its PA spin state of lowest energy during the whole simulation. In simulation \#37, after 30 orbits, the nucleus is rotating in a complex state with a relatively small spin period of about $6 \mathrm{~h}$. The initial spin period for this simulation is 10 days. In both simulations, the nucleus is considered uniformly active.

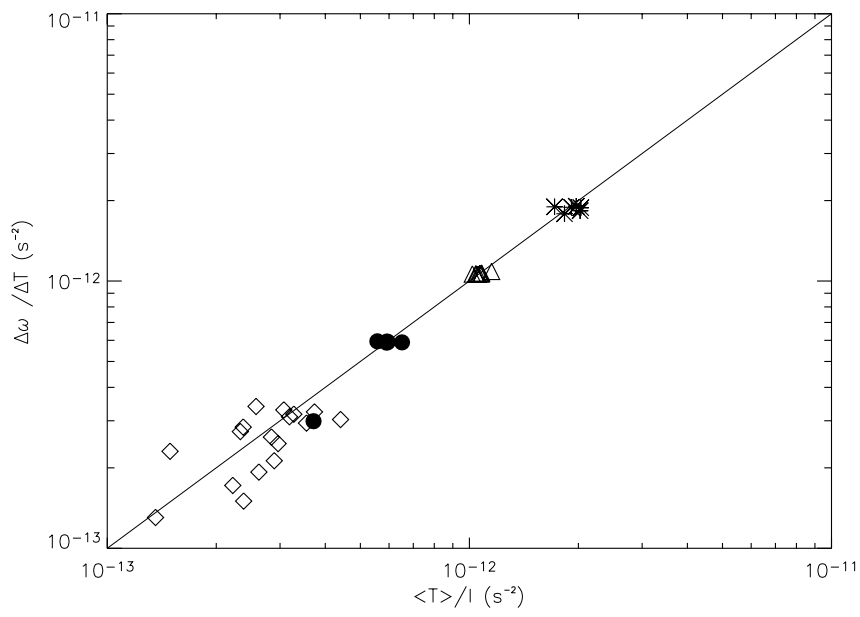

Fig. 9. Linear relationship between the change of the angular velocity $\omega$ and the mean value of the torque divided by the corresponding moment of inertia when the nucleus is in the PA spin state of lowest energy. The mean value of the torque component has been calculated in two steps. First, the instantaneous torque component has been filtered out in order to remove short-term variability. Second, it has been integrated in time along the total orbital path and normalized by the or-

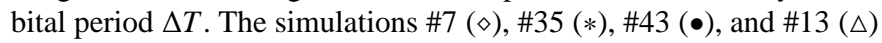
have been used. During the simulation \#35, the body rotates around the largest principal axis (PA spin state of maximum energy). In the other ones, the body rotates around the shortest principal axis (PA spin state of minimum energy). The points fit well the linear relationship of Samarasinha (1986).

small spin period and experiences a slow temporal evolution of the spin period (e.g., simulations \#23, \#25, and \#37). This happens when the spin period decreases after the nucleus got to a NPA spin state. This is the case in simulation \#37 (Fig. 8), in which the nucleus rotates in a NPA spin state with a mean spin period close to $6 \mathrm{~h}$ after 30 orbits.
The variations of the spin period occur in the timescale of the relationship (1) (Samarasinha et al. 1986). We made additional calculations in order to estimate the effective moment arm $\mathrm{d} r_{n}$ in Eq. (1) when the activity is distributed across the surface. According to this relationship (and to the Euler equations), the change of the main component of the angular velocity is equal to the ratio between the corresponding torque component and the moment of inertia.

In Fig. 9, we have compared the temporal variation of the angular velocity with the mean value of the component of the torque parallel to the angular momentum for simulations in which the nucleus is rotating close to a PA state (LAM or SAM). The linear relationship between these two quantities allows us to determine typical values for the effective moment arm coefficient $d$ used in Eq. (1). Using the water production rate at perihelion predicted by the model for $\dot{m}$, a typical value $v_{\mathrm{g}}=300 \mathrm{~m} / \mathrm{s}$ and $\eta=0.5$, and considering the moment of inertia and the mean nucleus radius $r_{n}$, we get $d \sim 0.01-0.04$ for the simulations shown in Fig. 9 (\#7, \#13, \#35, and \#43). We want to stress that this value is dependent on the orbit, on the orientation of the spin axis, on the distribution of activity and on the shape. The above range of values for $d$ must be regarded as typical for elongated nuclei of moderately active Jupiter-family comets.

There are two very different behaviors for the long-term evolution of the spin period, depending on how fast the direction of the angular momentum changes from one perihelion passage to the next.

When the orientation changes by less than 5-10 degrees per orbit ${ }^{4}$, the torque remains nearly constant from one orbit to the next. The long-term temporal evolution of the spin period can then be fitted by a hyperbolic function of time, in agreement with Eq. (1). This behavior is very frequent for simulations during which the nucleus remains rotating close to a PA spin state (e.g., simulations \#3, \#7 and \#15 of Figs. 4-6), but it has also been observed in some simulations in which the nucleus rotates in NPA spin state (e.g., simulations \#5 and \#17).

When the direction of the angular momentum changes very significantly (larger than 30 degrees) at each orbital step, the spin period evolves in a "random" way, even when the nucleus is in a PA state. This is the case for simulations \#18 (see Fig. 8), $\# 19$ and \#20. This "random" behavior is observed in the most elongated objects ( 3 and 4 in Fig. 1), when they are uniformly active. As the torque acting on these uniformly active nuclei is mainly driven by the illuminated surface, when the orientation of the spin axis changes significantly, the torque also changes and its effect can be totally different from an orbit to the next.

\subsection{Evolution of the direction of the angular momentum}

In all the simulations, we observe a displacement of the direction of the angular momentum. The amplitude of this displacement during one orbit depends strongly on the spin period, spin

4 This corresponds to a maximum rate of change at perihelion of $0.2 \mathrm{deg} /$ day. 


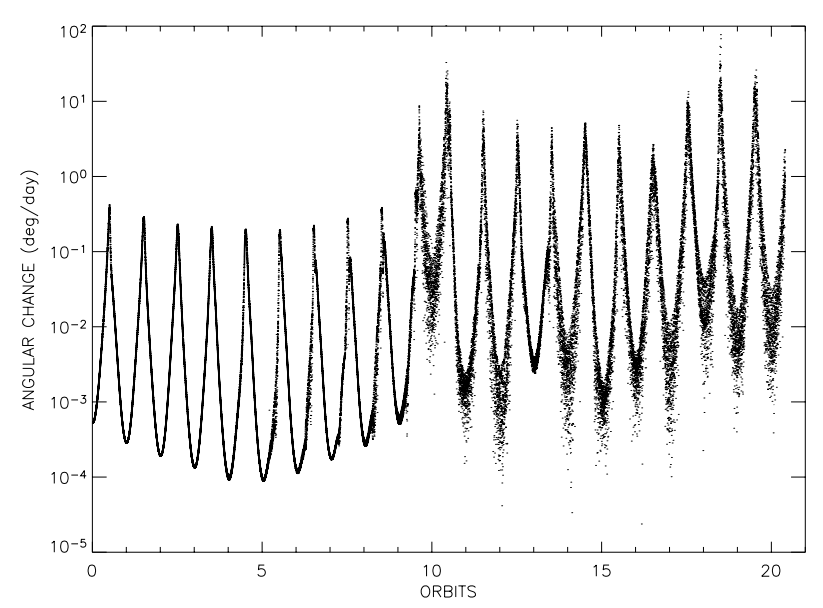

Fig. 10. Evolution of the daily angular velocity of the angular momentum direction in simulation \#9. In this simulation, the nucleus rotates close to its minimum energy level until the orbit \#10. At orbit \#10, the nucleus starts to rotate in a NPA spin state. Until the orbit \#10, the angular velocity is $\sim 0.1 \mathrm{deg} /$ day near perihelion. At $r_{\mathrm{h}} \sim 1.5-2.0 \mathrm{AU}$, the velocity decreases to $0.01 \mathrm{deg} / \mathrm{day}$, and at aphelion it becomes frequently lower than $0.001 \mathrm{deg} /$ day. The angular velocity increases to $\sim 1-10 \mathrm{deg} /$ day near perihelion when the nucleus gets to a NPA spin state. This translate into larger displacements from one perihelion passage to another.

state, size, shape and activity pattern of the nucleus at a given time.

For example, the maximum angular displacement for partially active nuclei is lower than $10 \mathrm{deg}$ when their spin period is $\sim 6 \mathrm{~h}$, and it becomes much lower when the spin period decreases. Uniformly active nuclei rotating close to a PA state have maximum angular displacements of 30-40 deg. The angular velocity associated to this displacement is $\sim 0.1-0.001 \mathrm{deg} /$ day (Fig. 10).

For comets rotating in NPA spin states, the direction of the angular momentum is shifted by larger angles from one passage to another and the angular velocity increases accordingly (see Fig. 10). The maximum angular displacement for partially active nuclei is $\sim 30 \mathrm{deg}$ when the spin period is close to $6 \mathrm{~h}$. For uniformly active nuclei, the maximum angular displacement is frequently larger that $50 \mathrm{deg}$ when they are rotating with a spin period around $6 \mathrm{~h}$, reaching values larger than $150 \mathrm{deg}$ when the spin period increases. Note that these values obviously depend on the parameters used in the model.

Samarasinha (2002) suggested that the direction of the angular momentum should follow a spiral which tends to be aligned to the direction of the NGF when the outgassing is maximum, usually nearly in the orbital plane. In our simulations, this spiral evolution toward the orbital plane is hardly seen. In Fig. 11, the evolution of the angular momentum direction is shown for two simulations. We have observed part of spirals in simulations with a small active area but in general we can not confirm that the direction of the angular momentum tends to be in the orbital plane.

The main differences compared to Samarasinha's (2002) simulations is that in our case the activity is distributed across the surface, and the nucleus has an irregular shape. Therefore, the direction of the NGF when the production rate is maximum can significantly change from one orbit to the next, and the ratio of the components of the torque varies during an orbital path. In order to explore the differences between the two approaches, we have performed some simulations with large ellipsoids with only one active cell, which allows to keep constant the ratio between the three components of the torque. We observe spiral-like structures showing an asymptotic evolution of the angular momentum toward the orbital plane, in agreement with Samarasinha's (2002) results. This preliminary analysis suggests that the spiral behavior toward the orbital plane could be restricted to nuclei with a very small or a very dominant active area. However, additional work needs to be done to explore in more detail how the direction of the angular momentum evolves as a function of time, and whether or not it moves toward a stable direction.

\subsection{Comparison with observations}

Like other authors before (e.g. Samarasinha et al. 1996; Neishtadt et al. 2002, etc.), we find that variations of the spin period can be caused by the torque of the NGF. A variation of the spin period of comet $\mathrm{P} /$ Tempel 2 has been detected at a 3- $\sigma$ level by Mueller \& Ferrin (1996). A variation of the spin period has also been observed in the long-period comet Levy 1990c. Schleicher et al. (1991) measured a spin period of $18.9 \pm 0.2 \mathrm{~h}$ when the comet was at $1.4 \mathrm{AU}$ pre-perihelion. Three weeks later, Feldman et al. (1992) reported a spin period of $17.0 \pm 0.1 \mathrm{~h}$. Interestingly enough, this variation took place in the same characteristic time (a few weeks) than in our simulations. Watanabe (1992) suggested that this spin-up could be explained by a shrinking of the nucleus. Nevertheless, his calculations showed that the density of the nucleus should be very low ( $\sim 0.14 \mathrm{~g} \mathrm{~cm}^{-3}$ for an $1-\mathrm{km}$ nucleus) to produce such a large spin-up. Large changes of the spin period of a kilometer-sized comet nucleus with a small active area and a mean initial spin period between 20 and $40 \mathrm{~h}$ in the preperihelion branch of the orbit are in agreement with our simulations.

Simulations also show that, for comets with a main active area, the long-term evolution of the spin period will have a hyperbolic variation (see Sect. 3.2). If such a variation could be observed, the moments of inertia of the nucleus could be estimated (and therefore its density if the nucleus size and elongation are known). However, that would imply the knowledge of the effective moment arm $d r_{n}$ defined in Eq. (1).

For such comets with restricted active areas, we also confirm that the direction of the angular momentum will spiral down to the orbital plane (Samarasinha 2002) ending up with a spin axis that has a high obliquity with respect to the orbital plane. Two comets fall possibly into this category: the spin axis of 19P/Borrelly has an obliquity of $\sim 100^{\circ}$ (Farnham \& Cochran 2002), and that of $109 \mathrm{P} /$ Swift-Tuttle is $\sim 80^{\circ}$ (Sekanina 1981b). For comets with activity patterns distributed across the surface, no such behavior has been clearly observed.

If the excitation occurs only after a transitory phase during which relationship (5) (Sect. 3.1) is verified, and assuming that the initial rotational state is a PA state of lowest 

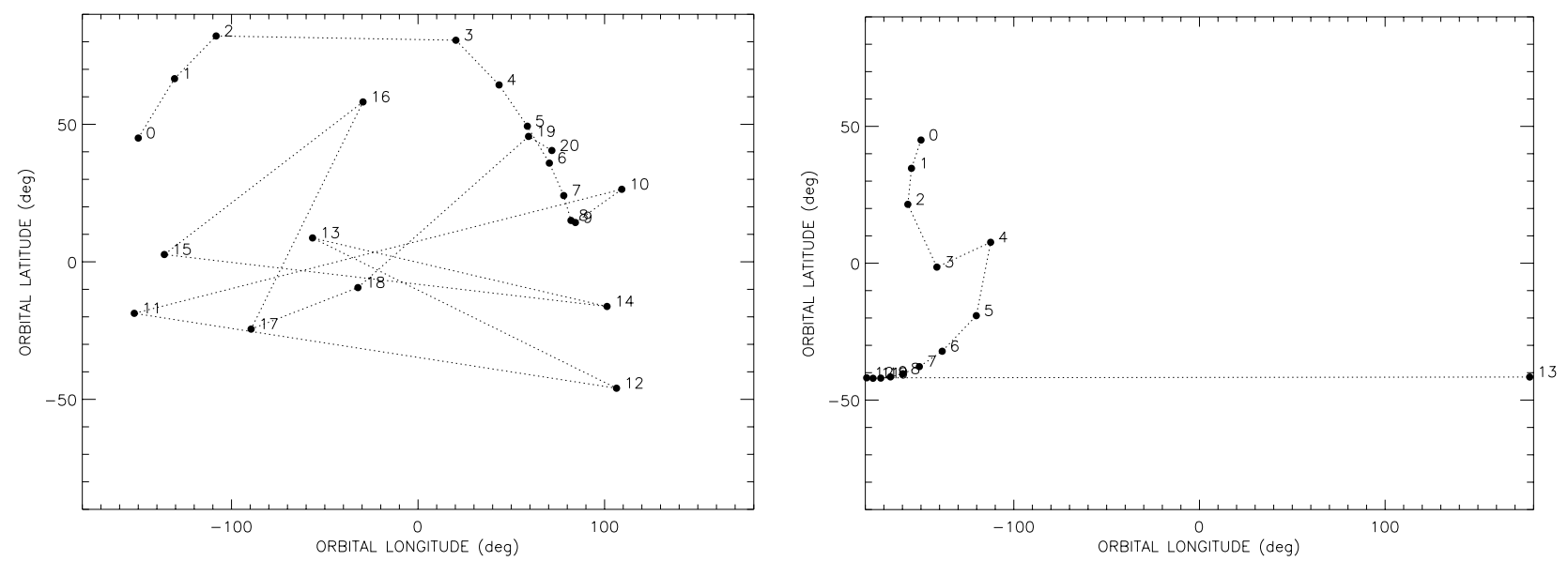

Fig. 11. The orientation of the angular momentum at aphelion for simulations \#9 (left) and \#15 (right). The simulation \#9 corresponds to the object 2 and a uniformly active surface. In this simulation the nucleus is rotating close to its PA state of minimum energy up to orbit \#10. It then starts to rotate in a NPA state. In this case a "random" evolution of the orientation of the angular momentum is observed. The simulation \#15 corresponds also to object 2 but with only $9 \%$ of its surface active. This nucleus is excited after orbit \#4 and remains rotating close to the PA state of maximum energy until the end of the simulation. After orbit \#4, the beginning of a spiral behavior can be seen.

energy, there are two populations of comets. The first population has NPA (LAM or SAM) spin states or it is rotating in the PA state of highest energy leaded by the torque. If $\left|N_{x}\right|$ and $\left|N_{z}\right|$ are comparable, these comets would have suffered a spinning down after they enter in the inner Solar System. The second population of comets is in the PA state of minimum energy or very close to it, either because relationship (5) has never been satisfied or because the nucleus returned to this state after having been temporarily excited. Small nuclei rather than large ones are more likely to belong to the first population because their spin period experiences changes in a shorter timescale according to the Eq. (1). However, not all of them belong to this population. At the moment, the observational constraints are insufficient to evaluate which proportion of comets is in the PA spin state of minimum energy and our limited set of numerical simulations do not allow us to make predictions on this point. However, from the simulations (bearing the parameters used in mind) active and small short-period comets might actually be in such a PA spin state.

\section{Summary and conclusions}

We have performed simulations of the long-term evolution of the spin state of small active comet nuclei in the orbit of 46P/Wirtanen under the effect of the torque of the NGF. A total of 46 combinations of irregular shapes and activity patterns have been simulated. In all of them, the three moments of inertia are different. A simplified thermophysical model has been used to compute the NGF and its torque. These simulations have allowed us to reach the following results and conclusions.

- We observe typical changes of the spin period of $0.01-10 \mathrm{~h}$ per orbit, depending on the initial spin period and on the excitation level. Significant changes (several hours) can occur near perihelion in less than a month. At the same time, typical changes of the direction of the angular momentum ranging from 0.1 to several tens of degrees per orbit frequently occur in the simulations.
- These changes are not always associated to an observable excitation of the spin state. The excitation process requires that the condition of Eq. (5) is fulfilled. This is equivalent to say that, for a triaxial body with comparable torque components, the "spin period" (as defined in Sect. 3) must be above a critical value when the excitation occurs. Nearlyprolate bodies are more easily excited than the asymmetric ones, in agreement with this relationship.

- Small and active comet nuclei are more likely excited than large inactive ones because their spin periods evolve in a shorter timescale. However, even a small active nucleus like 46P/Wirtanen could remain during tens of orbits in its PA state of minimum energy (pure spin).

- The activity pattern has a strong influence on the long-term evolution of the rotational parameters. When the activity is restricted to a main active area, the direction of the angular momentum tends to evolve in spiral toward the orbital plane. The evolution of the spin period is qualitatively the same from a perihelion passage to the next because the relative value of the three components of the torque remains constant. When these bodies get excited, they usually reach the PA spin state of maximum energy before the end of the simulation.

- In the opposite case, when the activity is distributed across the surface, the evolution of the rotational parameters frequently follows a "random" (unpredictable) evolution.

Repeated measurements of the spin period of a comet would be of great interest to constrain the value of its moments of inertia, and the density if the size and shape of the nucleus have been determined. It is desirable that these measurements are combined with measurements of the water production rate. The results will have consequences during the preparation of space missions to comets, especially Rosetta. They can also help us to interpret past and future groundbased data. Future simulations should allow to cover better the range of possible parameters (nucleus size and shape, activity pattern). 
Acknowledgements. PJG is currently supported by an ESA External Research Fellowship.

\section{References}

Belton, M. J. S. 1991, in Comets in the Post Halley era, ed. R. L. Newburn Jr, M. Newgebauer, \& J. Rahe (Dordrecht, The Netherlands: Kluwer Academic Press), 691

Bertaux, J. L., Costa, T., Mäkinen, E., et al. 1999, Planet. Space Sci., 47,725

Crifo, J. F. 1987, A\&A, 187, 438

Farnham, T. L., \& Cochran, A. L. 2002, Icarus, 160, 398

Feldman, P. D., Budzien, S. A., Festou, M. C., A'Hearn, M. F., \& Tozzi, G. P. 1992, Icarus, 95, 65

Fink, U., Hicks, M. D., Fevig, R. A., \& Collins, J. 1998, A\&A, 335, L37

Gutiérrez, P. J. 2001, Ph.D. Dissertation, Universidad de Granada

Gutiérrez, P. J., Ortiz, J. L., Rodrigo, R., \& López-Moreno, J. J. 2001, A\&A, 355, 326

Gutiérrez, P. J., Ortiz, J. L, Rodrigo, R., López-Moreno, J. J., \& Jorda, L. 2002, Earth Moon and Planets, 90, 239

Jewitt, D. C. 1992, Proc. of the 30th Liège International Colloq., 85

Jorda, L., \& Gutiérrez, P. 2002, Earth Moon and Planets, 89, 135

Julian, W. H. 1988, Icarus, 74, 377

Keller, H. U., Jorda, L., Rickman, H., \& Thomas, N. 2000, Amer. Astron. Soc., DPS Meet. \#32, \#36.11

Landau, L. D., \& Lifshitz, E. M. 1976, Mechanics, 3rd ed. (Pergamon Press)

Lamy, P. L., Toth, I., Jorda, L., et al. 1997, A\&A, 335, L25

Marsden, B. G., Sekanina, Z., \& Yeomans, D. K. 1973, AJ, 78, 211
Marsden, B. G., \& Williams, G. V. 1999, Cataloge of Comettary Orbits, 13th ed., IAU Minor Planet Center Smithsonian Astrophysical Observatory (Cambridge)

Mueller, B. E. A., \& Ferrin, I. 1996, Icarus, 123, 463

Muinonen, K. 1998, A\&A, 332, 1087

Neishtadt, A. I., Scheeres, D. J., Sidorenko, V. V., \& Vasiliev, A. A. 2002, Icarus, 157, 205

Peale, S. J., \& Lissauer, J. J. 1989, Icarus, 79, 396

Peltoniemi, J. I., Lummne, K., Muinonen, K., et al. 1989, Appl. Opt., 28, 4088

Rickman, H. 1989, Adv. Space Res., 9, (3)59

Rickman, H., \& Jorda, L. 1998, Adv. Space Res., 21, 1491

Rodionov, A. V., Crifo, J.-F., Szegö, K., Lagerros, J., \& Fulle, M. 2002, Planet. Space Sci., 50, 983

Samarasinha, N., A'Hearn, M. F., Hoban, S., \& Klinglesmith, D. A. 1986, in 20th ESLAB Symp. on the Exploration of Halley's comet, 27-31 Oct. 1986, Heidelberg, Germany, ESA-SP 250, vol. 1,487

Samarasinha, N., \& Belton, M. J. S. 1995, Icarus, 116, 340

Samarasinha, N., Mueller, B. E. A., \& Belton, M. J. S. 1996, Planet. Space Sci., 44, 275

Samaransinha, N. 2002, Proc. of the IAU Colloq. 168, Nanjing, China, Pub. Astron. Soc. Pacific, San Francisco, in press

Schleicher, D. G., Millis, R. L., \& Osip, D. J. 1991, Icarus, 94, 511

Sekanina, Z. 1981a, Ann. Rev. Earth Planet. Sci., 9, 113

Sekanina, Z. 1981b, AJ, 86, 1741

Szegö, K., Crifo, J. F, Földy, L., Lagerros, J. S. V., \& Rodionov, A. V. 2001, A\&A, 370, L35

Watanabe, J-I. 1992, Publ. Astron. Soc. Japan, 44, 163

Whipple, F. 1950, AJ, 111, 375

Wilhelm, K. 1987, Nature, 327, 27 